\title{
Repeated autologous umbilical cord blood infusions are feasible and had no acute safety issues in young babies with congenital hydrocephalus
}

\author{
Jessica M. Sun ${ }^{1}$, Gerald A. Grant ${ }^{2}$, Colleen McLaughlin', June Allison' ${ }^{1}$, Anne Fitzgerald' ${ }^{1}$, Barbara Waters-Pick' and \\ Joanne Kurtzberg'
}

BACKGROUND: Babies with congenital hydrocephalus often experience developmental disabilities due to brain injury associated with prolonged increased pressure on the developing brain parenchyma. Umbilical cord blood (CB) infusion has favorable effects in animal models of brain hypoxia and stroke and is being investigated in clinical trials of brain injury in both children and adults. We sought to establish the safety and feasibility of repeated intravenous infusions of autologous CB in young babies with congenital hydrocephalus.

METHODS: Infants with severe congenital hydrocephalus and an available qualified autologous CB unit traveled to Duke for evaluation and $\mathrm{CB}$ infusion. When possible, the $\mathrm{CB}$ unit was utilized for multiple infusions. Patient and CB data were obtained at the time of infusion and analyzed retrospectively.

RESULTS: From October 2006 to August 2014, 76 patients with congenital hydrocephalus received 143 autologous CB infusions. Most babies received repeated doses, for a total of two $(n=45)$, three $(n=18)$, or four $(n=4)$ infusions. There were no infusion-related adverse events. As expected, all babies experienced developmental delays.

CONCLUSION: Cryopreserved CB products may be effectively manipulated to provide multiple CB doses. Repeated intravenous infusion of autologous CB is safe and feasible in young babies with congenital hydrocephalus.

$\mathbf{H}$ ydrocephalus results from an excessive accumulation of cerebral spinal fluid (CSF) within the ventricular system of the brain resulting in a progressive increase in ventricular volume and intracranial pressure. It may be caused by a blockage within the ventricular system, overproduction of cerebral spinal fluid, or decreased absorption of cerebral spinal fluid.

The incidence of congenital hydrocephalus has been estimated as 0.3 to 1.5 per 1,000 live births $(1,2)$. Congenital hydrocephalus may occur in isolation or as a result of neural dysgenesis, such as spina bifida or a Dandy Walker malformation. Though many patients with congenital hydrocephalus are suspected to have a genetic cause, a causal mutation is identified in only a small portion of patients, most commonly X-linked hydrocephalus due to a mutation in L1CAM (3). Various other chromosomal abnormalities have been described in babies with hydrocephalus with additional associated somatic defects. Such genetic cases can usually be identified before or at birth.

Most cases of congenital hydrocephalus are diagnosed in utero on routine prenatal, screening ultrasonography when macrocephaly and/or ventriculomegaly are seen. Typically signs of hydrocephalus are first recognized between $15-10 \mathrm{wk}$ of gestation. Options at the time of an in utero diagnosis of severe hydrocephalus are currently limited to termination of the pregnancy or expectant management. Fetal shunt placement has been attempted, but technical issues with the shunt and mixed patient outcomes resulted in a moratorium on fetal percutaneous shunting in the United States since $1985(4,5)$.

Current standard management of a baby with severe congenital hydrocephalus involves aggressive monitoring of the third trimester of pregnancy and delivery when fetal lung maturity is achieved. Ventriculoperitoneal shunt placement is performed shortly after birth to divert the flow of CSF and decrease the intracranial pressure. In the modern era, shunt placement is associated with very low postoperative mortality, although there is still a moderate risk of infection in the neonatal period. A newer procedure called an endoscopic third ventriculostomy along with choroid plexus coagulation is now being performed in hopes of avoiding a shunt, although a shunt is still most common to manage severe hydrocephalus in the neonatal period. Survivors face the sequelae of both shunt complications and brain injury resulting from the prolonged hydrocephalic state in utero including motor deficits (50-60\%), auditory and visual deficits (25-60\%), seizures (20-50\%), and impaired intelligence (50-70\%) (6-13). Additional comorbidities may be present if a genetic syndrome is diagnosed postnatally or if other anatomical abnormalities are present on imaging, such as agenesis of the corpus callosum, colpcephaly, holoprosencephaly, or Dandy Walker malformation.

Umbilical cord blood (CB) has been shown to lessen the clinical and radiographic impact of hypoxic brain injury and stroke 
in animal models (14-18). In particular, Ballabh and colleagues have developed a model of intraventricular hemorrhage in rabbit pups that is followed by the development of hydrocephalus and subsequent white matter demyelination (19). In this model, intraventricular administration of human CB cells 24 and $72 \mathrm{~h}$ after injury failed to prevent the hydrocephalus, but did reduce subsequent demyelination (Ballabh, personal communication, 2014). CB has also been shown to engraft and differentiate in the brain, facilitating neural cell repair, in animal models and human patients with inborn errors of metabolism undergoing allogeneic, unrelated donor CB transplantation $(20,21)$. Intravenous infusion of autologous CB is currently under investigation for the treatment of acquired brain injuries including hypoxic ischemic encephalopathy (22), cerebral palsy (23), and spinal cord injury. A small safety trial testing CB-derived mesenchymal stem cells (MSCs) delivered directly into the airways of premature babies at risk for bronchopulmonary dysplasia has also been reported (24).

We have previously reported the safety of intravenous autologous CB infusion in 184 children with brain injury. The median age of infusion in that series was $2 \mathrm{y}$, and most children received a single infusion (23). Building on this experience, we hypothesized that autologous CB infusion might facilitate repair of the brain subjected to the pressure injury caused by severe congenital hydrocephalus. Prenatal diagnosis of these infants and delivery by planned C-section allowed for optimization of CB collection and banking at birth. The small size of the baby relative to the number of cells harvested in a typical $\mathrm{CB}$ collection allowed for planning of administration of more than one dose of $\mathrm{CB}$ during the first 1-2 y of life. The purpose of this investigation was to determine the safety and feasibility of repeated doses of autologous CB given intravenously to very young infants with brain injury due to severe congenital hydrocephalus.

\section{RESULTS}

\section{Patients}

Between October 2006 and August 2014, 76 patients with congenital hydrocephalus were treated with 143 autologous CB infusions. The most common etiology for hydrocephalus was aqueductal stenosis (46\%). Four patients were subsequently diagnosed with genetic conditions: one each with Aicardi syndrome, Walker Warburg syndrome, and a partial duplication and interstitial deletion of chromosome 6, and one patient with both a partial deletion of chromosome 10 and a partial duplication of the $\mathrm{X}$ chromosome. See Table 1 for patient characteristics.

\section{CB Units (CBUs)}

CBUs were collected at the time of delivery by the mother's obstetrical provider or, when available, trained collectors from the Carolinas Cord Blood Bank, a public CB bank housed at Duke University. Fifty-six (74\%) units were stored as directed donors. Of these, nine patients were born and had CBUs collected at Duke and 47 (84\%) were collected remotely via a kit program and then shipped to the Duke Stem Cell Lab for processing and
Table 1. Patient characteristics

$(n=76)$

\begin{tabular}{lc}
\hline Age at first infusion & \\
Median & 2 mo \\
Range & 6 d to $4.5 y$ \\
Gender $(N, \%)$ & \\
Male & $39(51 \%)$ \\
Female & $37(49 \%)$ \\
Diagnosis ( $N, \%)$ & \\
Aqueductal stenosis & $35(46 \%)$ \\
Stroke/hemorrhage & $10(13 \%)$ \\
$\quad$ Other (Dandy-Walker variant, Chiari ll without spina & $31(41 \%)$ \\
bifida, etc) & \\
\hline
\end{tabular}

storage. The remaining 20 (26\%) CBUs were stored at six different private CB banks (four US, two international). For units in which it was recorded $(n=68)$, the median collection volume of CB was $55 \mathrm{ml}$ (range 5-180 ml). When these units were identified as potential candidates for infusion, low resolution HLA typing was performed on both a test sample of the CBU and the patient for identity confirmation. Almost all CBUs $(n=73$, 96\%) were stored in two-compartment bags. When possible, only one compartment or a portion of one compartment was utilized for infusion to provide an adequate cell dose, allowing the remainder to be stored for later infusions. The median precryopreservation total nucleated cell count (TNCC) of the CBUs was $4.81 \times 10^{8}$ (range $0.15-18.6 \times 10^{8}$ ), median viability $97 \%$ (range 71-100\%). Despite negative precryopreservation sterility cultures, five CBUs had positive post-thaw cultures (coagulase negative Staphlococcus (4), Streptococcus viridans (1)). One of the positive cultures was on a third infusion from a CBU from which prior post-thaw cultures on the first and second infusions were negative. Since these culture results were not available until 24-72 h after the infusions, no patients were treated with antibiotics. At the time the positive culture was reported, a staff member communicated with the patient's parents to determine whether there was any concern for post infusion infection. No patient had concerning symptomatology and no antibiotic treatment was initiated. There were no clinical infections documented in any patient.

When the TNCC allowed, a portion of the CBU was utilized for infusion. If a CBU was stored in a bag with an 80/20 configuration, the $20 \%$ compartment was generally used for the first infusion. If the $80 \%$ compartment contained an TNCC of $>5 \times 10^{7} / \mathrm{kg}$ at the time of the second infusion, then only a portion of the cells in the $80 \%$ compartment were used and the remaining thawed cells were refrozen in an 80/20 bag for future dosing. In this study, 19 infusions were performed using cells that had been previously thawed and refrozen. The median post-thaw recovery of TNCC of the initial thaws of these products was 66\% (range 41-78\%). When these units were refrozen and subsequently rethawed, the median post-thaw recovery of TNCC was 74\% (range 50-109\%) of the refrozen cells and $47 \%$ (range 21-72\%) of the initial TNCC. As many of these CBUs 
were retrieved from private $\mathrm{CB}$ banks, they were not tested for CD34 content and colony forming units (CFUs) prior to cryopreservation, making it impossible to compare recovery after the initial thaw. After refreezing, the median recovery of CFUs was $64 \%$ (range $12->100 \%$ ) and median recovery of CD34+ cells was $52 \%$ (range $0->100 \%$ ) compared to CFUs and CD34+ cells obtained at the time of the initial thaw.

\section{Infusions}

The median age at the time of the first infusion was 2 mo (range $6 \mathrm{~d}$ to $4.5 \mathrm{y}$ ). Most babies received repeated doses, for a total of two $(n=45)$, three $(n=18)$, or four $(n=4)$ infusions, as per the timeline in Figure 1. Median cell doses per infusion were TNC $1.9 \times 10^{7} / \mathrm{kg}$ (range $0.1-13.3 \times 10^{7} / \mathrm{kg}$ ) and CD34 dose $0.5 \times 10^{5} / \mathrm{kg}$ (range $0-6.4 \times 10^{5} / \mathrm{kg}$ ); cell doses are listed by infusion number in Table 2 . All babies were premedicated before each infusion with a single dose of diphenhydramine $(0.5 \mathrm{mg} /$ $\mathrm{kg} \mathrm{IV})$, acetaminophen (10 mg/kg PO), and methylprednisolone $(0.5 \mathrm{mg} / \mathrm{kg} \mathrm{IV})$. The infusions were well tolerated, with no acute or long-term adverse reactions noted.

\section{Patient Follow-Up After CB Infusion}

The overall survival of the patients in this study was $97 \%$ with a median follow-up of $1.1 \mathrm{y}$ (range 0.1-7.5 y). Two patients died from issues unrelated to the CB infusion; one 4 mo after infusion due to complications from a surgical craniosynastosis repair, and one who died in his sleep 2 mo after infusion. As expected, all infants had motor delays in the first year of life due to increased head size relative to the baby's height and weight. At the time of last follow-up, $42 \%$ of patients had been diagnosed with seizures and $52 \%$ of patients had persistent vision and/or hearing impairments.

\section{DISCUSSION}

In this report, we describe our initial clinical experience giving multiple intravenous autologous CB infusions in a heterogeneous group of infants with congenital hydrocephalus. As this was a phase I, first-in-man, proof-of-concept safety study, there was no control group. Since efficacy of cell therapy for congenital hydrocephalus is unknown, safety was of utmost importance. We therefore chose to use banked CB, a cell therapy product that has already had a favorable safety profile in the clinic. In addition, given the very young age of these patients, we felt strongly that an autologous product would pose the lowest risk. Furthermore, theoretical concerns have been raised

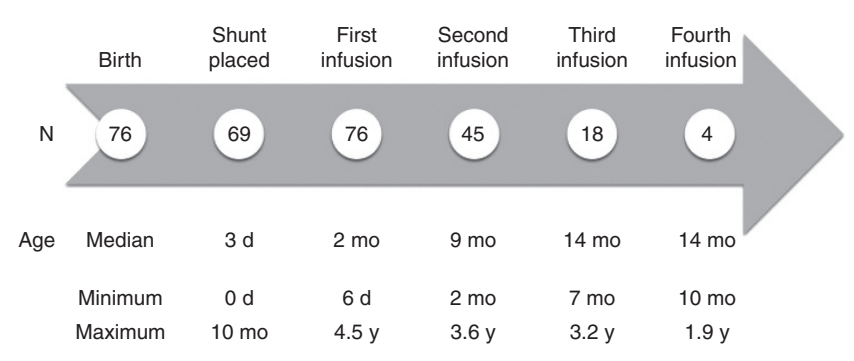

Figure 1. Timeline of shunt placement and cord blood infusions. about inducing aberrant immune tolerance to donor antigens (HLA or other) if young babies are exposed to third party cells during the early phases of immune ontogeny. Dimethyl sulfoxide and dextran are utilized during cryopreservation of $\mathrm{CB}$, and dextran and human serum albumin are utilized during thawing and washing. Thus, the babies in this series were exposed to residual amounts of these excipients with each infusion. Prior to this study, only a few dozen infants $<3$ mo of age had been treated with allogeneic, banked CB for correction of genetic diseases, and there was no prior experience giving cryopreserved and thawed products in single or multiple infusions to neonates and young infants. In this series, there were no acute or longterm adverse events related to $\mathrm{CB}$ infusion, indicating that the procedure is safe and feasible in these very young babies.

Due to the young age and small size of the patients, most (70\%) CBUs were large enough to provide more than one dose of cells and $24 \%$ of patients received three or more doses. The safety and feasibility of multiple CB infusions were also favorable with no increase in infusion reactions or later toxicities after subsequent infusions. It was feasible to manipulate $\mathrm{CB}$ products stored in dual compartment bags so that two to four doses could be administered at distinct and separate time points, and products that underwent recryopreservation and thawing demonstrated adequate cell recovery.

As the primary focus of this study was safety and feasibility, a control group was not included. Therefore, a treatment effect cannot be established. The efficacy of this approach requires additional investigation and continues to be challenging. Congenital hydrocephalus is a rare condition, and affected babies may have other associated abnormalities or genetic syndromes. The causes of congenital hydrocephalus are heterogeneous and, not surprisingly, affected babies experience a wide variability of outcomes that are not predictable based on early clinical or radiographic characteristics. Therefore, assessing the efficacy of $\mathrm{CB}$ infusion on the natural history of the disease remains challenging. There is also a variable response to shunting in this population both on imaging and predicting the expansion of the cortical mantle and correlating this with overall cognitive development.

There is increasing animal and human data to suggest that CB may have a role in the treatment of brain injuries. In a recent phase I trial at Duke University, 23 babies who sustained hypoxic ischemic encephalopathy at the time of birth were

Table 2. Cell doses per infusion

\begin{tabular}{|c|c|c|c|c|}
\hline & $\begin{array}{c}\text { First } \\
(n=76)\end{array}$ & $\begin{array}{l}\text { Second } \\
(n=45)\end{array}$ & $\begin{array}{l}\text { Third } \\
(n=18)\end{array}$ & $\begin{array}{l}\text { Fourth } \\
(n=4)\end{array}$ \\
\hline \multicolumn{5}{|c|}{ Post-thaw total nucleated cell count dose $\left(\times 10^{7} / \mathrm{kg}\right)$} \\
\hline Median & 1.95 & 2.08 & 1.15 & 0.56 \\
\hline Minimum & 0.25 & 0.25 & 0.13 & 0.29 \\
\hline Maximum & 13.30 & 5.68 & 2.40 & 3.56 \\
\hline \multicolumn{5}{|c|}{ Post-thaw CD34 dose $\left(\times 10^{5} / \mathrm{kg}\right)$} \\
\hline Median & 0.50 & 0.70 & 0.25 & 0.20 \\
\hline Minimum & 0.05 & 0.04 & 0.01 & 0.10 \\
\hline Maximum & 6.40 & 4.90 & 2.00 & 0.40 \\
\hline
\end{tabular}


treated with a standard cooling protocol and also received intravenous autologous CB infusions (22). CB was processed and infused fresh, without cryopreservation, in one to four doses within the first $72 \mathrm{~h}$ of life. Infusions were found to be safe in these critically ill babies, and babies receiving cells had increased survival rates to discharge (100 vs. $85 \%, P=0.20)$ and improved function at $1 \mathrm{y}$ of age (74 vs. $41 \%$ with development in the normal range, $P=0.05$ ) compared to a concomitant group of babies who were treated with cooling but did not receive cells. A phase II randomized trial is currently in development.

In older children with cerebral palsy, Korean investigators compared three groups of children: those who received allogeneic $\mathrm{CB}$ and erythropoietin, placebo $\mathrm{CB}$ and erythropoietin, and both placebos (25). They reported greater improvements in cognitive and select motor functions in children who received $\mathrm{CB}$ and erythropoietin vs. either control group. While there was no CB-only group for comparison, their findings are encouraging and should be replicated. Our group is also currently conducting a phase II randomized, double-blind, placebo-controlled, crossover study of intravenous autologous $\mathrm{CB}$ infusion in children ages 1-6 y with cerebral palsy. This study is expected to conclude in 2015.

The mechanism by which CB cells may potentially improve the outcome of brain injuries are multiple. In the acute setting, such as babies with hypoxic ischemic encephalopathy, CB cells may have the ability to deliver trophic factors that can provide anti-inflammatory and neuroprotective effects and enhance the survival potential of host cells. In the chronic setting, such as older children with cerebral palsy, CB cells may be able to increase the plasticity of the injured brain by enhancing synaptogenesis and angiogenesis, stimulating endogenous repair mechanisms, and/or inducing migration and proliferation of endogenous neural stem cells. In these scenarios, long-term engraftment of $\mathrm{CB}$ cells should not be required, raising the possibility of utilizing allogeneic products. It is not yet clear from either animal models or human studies if an ideal or maximum therapeutic window for intervention with cell therapy exists after brain injury. If one does, it is likely to vary by the type of injury and the age of the patient.

If cell therapies, including $\mathrm{CB}$, are proven to have a role in the treatment of neurologic injuries, the parameters of their use will certainly require further refinement. Many issues remain unknown, including ideal cell source, route of administration, dose and dosing regimen, timing, and role of immunosuppression. If the intent is to modulate host repair mechanisms, a multiple dosing regimen could be more effective than a single dose. Although the optimal cell dose is unknown, this case series demonstrates that a single CBU can yield sufficient cells for multiple intravenous doses in young children, and that repeated dosing is safe and feasible in babies with brain injuries. Data regarding the functional outcomes of babies with congenital hydrocephalus treated with CB infusion(s) are being collected under a separate IRBapproved protocol. Based on the favorable safety profile and feasibility of $\mathrm{CB}$ infusion in this population, a phase II trial in children with severe hydrocephalus is under development. In that study, diligent genetic screening and review of brain imaging will be necessary to exclude patients with identifiable genetic conditions and brain malformations. Additional trials using multiple dosing regimens are also planned for babies with hypoxic ischemic encephalopathy and children with cerebral palsy and autism.

\section{METHODS}

This analysis is a retrospective data review of patients treated with intravenous autologous CB infusion for congenital hydrocephalus by the Duke Pediatric Blood and Marrow Transplant Program. A waiver of authorization to conduct this study was approved by the Duke University Medical Center Institutional Review Board. At least one parent signed routine hospital consent for treatment as well as consent for data to be collected and shared with the National Marrow Donor Program and Center for International Blood and Marrow Transplant Research (NMDP/CIBMTR) for entry into the Stem Cell Transplant Outcomes Database. Charts of children infused from $3 / 2004$ to $8 / 2014$ were reviewed.

\section{Patients}

Infants with severe congenital hydrocephalus, diagnosed either before or after the child's birth, were either self-referred or referred by their treating physicians. Infants were eligible for autologous CB infusion if their parents elected to bank their $\mathrm{CB}$ at birth and if the $\mathrm{CB}$ met certain technical specifications enumerated below. Patients with known genetic diseases, brain malformations, spina bifida, ineligible CBUs, or inability to travel to Duke, were excluded.

\section{Treatment Plan}

If referred prenatally, information regarding the patient's diagnosis and expected date of delivery was acquired. CB collection was then arranged through the Stem Cell Lab, a clinical hospital laboratory at Duke University, via a directed donor kit collection program. If referred after birth and if the parents had elected to bank the baby's $\mathrm{CB}$ with a private $\mathrm{CB}$ bank, information regarding the patient's diagnosis, current condition, and CBU characteristics was obtained. In either case, donor screening labs were obtained on a maternal blood sample drawn around the time of delivery. After the child and their CBU were deemed eligible, identity and potency of the CB unit were confirmed. For units banked at private banking facilities, the CBU was shipped to the Duke Stem Cell Lab in a dry shipper maintaining temperatures $<-150^{\circ} \mathrm{C}$, processed on a Sepax 1 to volume reduce and partially red blood cell and plasma deplete, mixed with dimethyl sulfoxide/Dextran to a final concentration of $10 \%$ dimethyl sulfoxide, cryopreserved by controlled-rate freezing and stored under liquid nitrogen until the time of infusion. Some parents elected to have their babies born at Duke, so that they were able to have their CB collected and receive neonatal care including their initial neurosurgery at Duke. All patients not born at Duke traveled with their parent(s) to Duke for a 3-d visit including: on day 1, a baseline history, physical, and laboratory evaluation including donor screening labs (if the baby was $>30 \mathrm{~d}$ of age); on day 2, infusion of the autologous CB; and on day 3 , follow-up by phone to screen for infusion-related toxicities.

\section{CBU Criteria}

Cryopreserved CBUs had to meet the following minimum criteria for infusion as documented by the bank of origin: precryopreservation TNCC documented and $>1 \times 10^{7}$ cells $/ \mathrm{kg}$ calculated for the child's current body weight, sterility cultures performed and negative, maternal infectious history screen and infectious disease markers (minimally HIV 1 and 2, HTLV 1 and 2, Hepatitis B and C, CMV, West Nile virus, and syphilis) performed and negative. If not stored by the Duke STCL, in addition to meeting the above specifications, CBU identity was confirmed via HLA typing of both a test sample of the CBU and peripheral blood of the patient, and a test sample of the $\mathrm{CBU}$ was thawed and tested for viability, CFU, and CD34 to confirm potency before the baby was scheduled for an infusion. 


\section{CBU Thawing and Infusion Procedure}

Cryopreserved CBUs were thawed and washed as described by Rubinstein et al. (26) and resuspended in dextran . $_{40}+5 \%$ human serum albumin solution on the day of infusion. Thawed CBUs were tested for enumeration of TNCC, viable CD $34^{+}$cells, CFUs, cell viability via trypan blue, and sterility cultures. On the day of infusion, patients were admitted to the Duke Children's Health Center Day Hospital and IV access was established via a peripheral vein. After premedication with Tylenol $(10 \mathrm{mg} / \mathrm{kg} \mathrm{PO})$, Benadryl $(0.5 \mathrm{mg} / \mathrm{kg} \mathrm{IV})$, and Solumedrol $(0.5 \mathrm{mg} / \mathrm{kg} \mathrm{IV})$, patients received either a portion of or their entire CBU via peripheral IV infusion over 5-15 min. The volume of the infusion was adjusted postwash to deliver no more than $1.25 \mathrm{cc} / \mathrm{kg}$ over $15 \mathrm{~min}$. Intravenous fluids were administered at 1.5 times maintenance for $2-4 \mathrm{~h}$ after the CB infusion. Vital signs and pulse oximetry were monitored continuously during the infusion and every $30 \mathrm{~min}$ for $1-2 \mathrm{~h}$ postinfusion as medically indicated.

\section{Dosing and Multiple Infusions}

The target dose per infusion was $1-5 \times 10^{7}$ cells per kilogram of patient body weight at the time of infusion. Based on the configuration in which each CBU was stored and the number of cells available, only one compartment of the CBU bag or a portion of one compartment was utilized for infusion to provide an adequate cell dose, and the remainder was stored under liquid nitrogen for later infusions. Generally, when the CB unit was stored in a bag with the $80 / 20$ configuration, the smaller compartment was utilized for the first infusion. At the time of the second infusion, the $80 \%$ compartment was thawed and a portion of the thawed product was administered to deliver a targeted cell dose which was calculated based on the baby's weight. The remaining cells were recryopreserved in a new $80 / 20$ bag to allow for administration of additional 1-3 future doses. The exact number of doses available depended on the TNCC of the initial collection and the weight of the baby over time. When the CBU cell dose was sufficient for multiple infusions, patients received subsequent doses at intervals of approximately 2-6 mo as feasible based on their medical condition and feasibility of travel logistics.

\section{Data Collection and Statistics}

Data regarding patients (diagnosis, age, birth history, surgical history, developmental trajectory, and symptoms at birth and at the time of infusion), infusions, and autologous CBU characteristics (collection volume, precryopreservation TNCC, viability, sterility, CD34 and CFU as well as post-thaw TNCC, CD34 count, viability, sterility cultures, and post-thaw CFUs) were obtained from both a prospectively maintained clinical database and retrospective review of routine medical records. Descriptive statistics were calculated for $\mathrm{CBU}$ parameters.

\section{STATEMENT OF FINANCIAL SUPPORT}

This study was supported by The Julian Robertson Foundation, New York City, NY.

Disclosures: The authors to not have any conflicts of interest or financial ties to disclose.

\section{REFERENCES}

1. Garne E, Loane M, Addor MC, Boyd PA, Barisic I, Dolk H. Congenital hydrocephalus-prevalence, prenatal diagnosis and outcome of pregnancy in four European regions. Eur J Paediatr Neurol 2010;14:150-5.

2. Persson EK, Hagberg G, Uvebrant P. Hydrocephalus prevalence and outcome in a population-based cohort of children born in 1989-1998. Acta Paediatr 2005;94:726-32.

3. Tully HM, Dobyns WB. Infantile hydrocephalus: a review of epidemiology, classification and causes. Eur J Med Genet 2014;57:359-68.

4. Clewell WH, Johnson ML, Meier PR, et al. A surgical approach to the treatment of fetal hydrocephalus. N Engl J Med 1982;306:1320-5.
5. Manning FA, Harrison MR, Rodeck C. Catheter shunts for fetal hydronephrosis and hydrocephalus. Report of the International Fetal Surgery Registry. N Engl J Med 1986;315:336-40.

6. Hoppe-Hirsch E, Laroussinie F, Brunet L, et al. Late outcome of the surgical treatment of hydrocephalus. Childs Nerv Syst 1998;14:97-9.

7. Heinsbergen I, Rotteveel J, Roeleveld N, Grotenhuis A. Outcome in shunted hydrocephalic children. Eur J Paediatr Neurol 2002;6:99-107.

8. Erickson K, Baron IS, Fantie BD. Neuropsychological functioning in early hydrocephalus: review from a developmental perspective. Child Neuropsychol 2001;7:199-229.

9. Dalen K, Bruarøy S, Wentzel-Larsen T, Laegreid LM. Intelligence in children with hydrocephalus, aged 4-15 years: a population-based, controlled study. Neuropediatrics 2008;39:146-50.

10. Kirkinen P, Serlo W, Jouppila P, Ryynanen M, Martikainen A. Long-term outcome of fetal hydrocephaly. J Child Neurol 1996;11:189-92.

11. Sato O, Yamguchi T, Kittaka M, Toyama H. Hydrocephalus and epilepsy. Childs Nerv Syst 2001;17:76-86.

12. Idowu OE, Balogun MM. Visual function in infants with congenital hydrocephalus with and without myelomeningocoele. Childs Nerv Syst 2014;30:327-30.

13. Ricci D, Luciano R, Baranello G, et al. Visual development in infants with prenatal post-haemorrhagic ventricular dilatation. Arch Dis Child Fetal Neonatal Ed 2007;92:F255-8.

14. Vendrame M, Cassady J, Newcomb J, et al. Infusion of human umbilical cord blood cells in a rat model of stroke dose-dependently rescues behavioral deficits and reduces infarct volume. Stroke 2004;35:2390-5.

15. Chen J, Sanberg PR, Li Y, et al. Intravenous administration of human umbilical cord blood reduces behavioral deficits after stroke in rats. Stroke 2001;32:2682-8.

16. Willing AE, Lixian J, Milliken $M$, et al. Intravenous versus intrastriatal cord blood administration in a rodent model of stroke. J Neurosci Res 2003;73:296-307.

17. Taguchi A, Soma T, Tanaka H, et al. Administration of CD34+ cells after stroke enhances neurogenesis via angiogenesis in a mouse model. J Clin Invest 2004;114:330-8.

18. Meier C, Middelanis J, Wasielewski B, et al. Spastic paresis after perinatal brain damage in rats is reduced by human cord blood mononuclear cells. Pediatr Res 2006;59:244-9.

19. Chua CO, Chahboune $\mathrm{H}$, Braun $\mathrm{A}$, et al. Consequences of intraventricular hemorrhage in a rabbit pup model. Stroke 2009;40:3369-77.

20. Escolar ML, Poe MD, Provenzale JM, et al. Transplantation of umbilical-cord blood in babies with infantile Krabbe's disease. N Engl J Med 2005;352:2069-81.

21. Kurtzberg J, Kosaras B, Stephens C, Snyder EY. Umbilical cord blood cells engraft and differentiate in neural tissues after human transplantation. Biol Blood Marrow Transplant 2003;9:128-9.

22. Cotten CM, Murtha AP, Goldberg RN, et al. Feasibility of autologous cord blood cells for infants with hypoxic-ischemic encephalopathy. J Pediatr 2014;164:973-979.e1.

23. Sun J, Allison J, McLaughlin C, et al. Differences in quality between privately and publicly banked umbilical cord blood units: a pilot study of autologous cord blood infusion in children with acquired neurologic disorders. Transfusion 2010;50:1980-7.

24. Chang YS, Ahn SY, Yoo HS, et al. Mesenchymal stem cells for bronchopulmonary dysplasia: phase 1 dose-escalation clinical trial. J Pediatr 2014;164:966-972.e6.

25. Min K, Song J, Kang JY, et al. Umbilical cord blood therapy potentiated with erythropoietin for children with cerebral palsy: a double-blind, randomized, placebo-controlled trial. Stem Cells 2013;31:581-91.

26. Rubinstein P, Dobrila L, Rosenfield RE, et al. Processing and cryopreservation of placental/umbilical cord blood for unrelated bone marrow reconstitution. Proc Natl Acad Sci USA 1995;92:10119-22. 